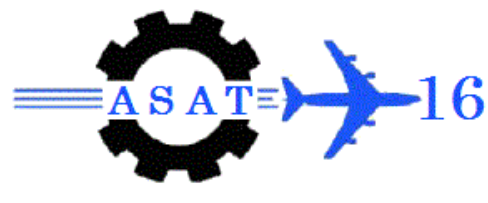

\title{
Estimation of PN Code of DS-CDMA Signals over Frequency selective Fading Channel in presence of Impulsive noise
}

By

Ahmed Fathy Darwesh*, Dr. Gamal Eldin M.Hamad** ,Prof. Dr. Ahmed Elsayed Elmahdy+

\section{Abstract:}

Spread spectrum signals are widely used for secure communications, as well as for multiple access interference (MAI). They have many interesting properties, including low probability of interception. Indeed, direct sequence spread spectrum systems (DSSSS) use a periodical pseudo-random sequence to modulate the baseband signal before transmission. Interception of DSSS signals is a difficult task due to the hiding of the PN sequence, especially if the accompanied noise is non-Gaussian and the channel is frequency selective.

In this paper, a receiver is proposed to intercept asynchronous direct sequence code division multiple access (DS-CDMA) signals received from different users and estimate the PN sequences of each one of them. The signals are assumed to be transmitted over frequency selective fading channel and contaminated with Class-A impulsive noise. The proposed interception receiver is based on Independent Component Analysis (ICA), the pseudo random sequences is estimated from the eigenvector subspace in case of single path. In multipath situations, the estimation is the overlapped form of the pseudo random sequences, a triple correlation function (TCF) is introduced to eliminate the influence of multipath. The performance of proposed receiver is evaluated in terms of the number of correct estimated codes for m-sequence over typical urban channel in presence of impulsive noise. The results show that the interception receiver performs is $100 \%$ code estimation at $-8 \mathrm{~dB}$ signal to noise ratio.

Keywords: PN sequence; DS-CDMA; m-sequence; Impulsive noise; frequency selective fading channel; ICA; TCF.

\section{Introduction}

Direct Sequence Spread Spectrum (DSSS) communication has many characteristics, such as wide spectrum, low power spectral density, confidentiality, anti-interference and easy to use in multiple access communication. Combined with the double merits of multiple access and direct sequence spread spectrum, direct sequence code division multiple access (DS-CDMA) has been widely used in both military and civil communications. The PN sequences are very important parameters in DS-CDMA communication system, from which multi-user can share the same frequency band at the same time [1].

\footnotetext{
* Egyptian Armed Forces, a.fathymtc@yahoo.com

$* *$ Egyptian Armed Forces

+ German University in Cairo
} 
At present, the researchers has conducted in-depth study of blind estimation of the PN sequence of DSSS signal, and put forward a series of methods, such as in [2], this method proposed a cipher analysis method of $\mathrm{m}$-sequence utilizing the logic character of linear feedback shift register (LFSR). This method made use of only 1th order statistic therefore it was sensitive to noise. In [3] the previous method has adopted with maximum vote criterion to find the primitive polynomial of $\mathrm{m}$-sequence. When information modulation existed, the method was not available any more. In [4] it is proposed a method of pseudo random estimation based on principal component analysis (PCA), extended the method to the situation of two users in [5], and analysis the performance from the idea of eigenvalue decomposition (EVD) in [6]. But it was not available in multiuser situations. The study of blind estimation of PN sequences of DS-CDMA signal was started later, and had fewer achievements. In DS-CDMA system, information sequences of different users remained statistically independent and the pseudo random sequences of different users remained none correlation, so the DS-CDMA signals were fit for the theory model of blind source separation (BSS) [7]. ICA, the typical technology of BSS, therefore could be used to achieve the blind separation of DS-CDMA signals in non-cooperative situations, only when few parameters were estimated. With the development of DS-CDMA signal processing technology, some efficient methods of parameter estimation for the carrier frequency [8], chip interval [9], and symbol interval [10] of the DS-CDMA signals had appeared. In [11] it is used ICA method to detect the information of DS-CDMA system blindly. If the period of PN sequence was known, we could get the information sequence of each user from asynchronous DS-CDMA signal using ICA, and estimating the true PN code for each user.

From the above survey we note that researchers have not focused on blind estimation of PN sequence over multipath channel in presence of impulsive noise, although most of wireless communication systems work on multipath channel and typically wireless transceiver designs are based on the assumption that noise is additive, white and Gaussian (AWG) [12]. These transceivers perform fine in normal environments (where optimum reception can be achieved with Gaussian channel assumption) but their applicability in noise intensive electricity substation environment is not risk free and needs thorough investigation [13]. Partial discharges is the major source of impulsive noise, in electricity transmission substations and if the risks of deploying wireless communications equipment are to be properly assessed the impact of such impulsive processes requires thorough evaluation [14]. Accordingly, a method based on ICA was proposed here to achieve the estimation of overlapped PN sequences because of multipath and by using TCF we can get the true sequence for each user. Simulation results proved the validity of the method proposed here and give a good performance under high effects of impulsive noise and multipath channel.

The rest of the paper is organized as follows: In Section II, mathematical model is presented. The analysis method of ICA and TCF are introduced Section III. Simulation results are presented in Section IV.

\section{SYSTM MODEL}

A. Asynchronous DS-CDMA Model

In asynchronous DS-CDMA, each of the $K$ users uses his own m-sequence to spread his narrowband information signal. Consider a BPSK modulation the received baseband signal can be written as:

$$
S(t)=\sum_{k=1}^{K} S_{k}(t)
$$

where $S_{\mathrm{k}}(\mathrm{t})$ is the $K^{\text {th }}$ user's transmitted signal, its complex baseband representation could be modeled as: 


$$
S_{k}(t)=\sqrt{\xi_{k}} \sum_{l=1}^{l_{k}} \sum_{m=1}^{N} h_{k, l} b_{k, m} C_{k}\left(t-m T_{b}-\tau_{k, l}\right)
$$

where $\xi_{\mathrm{k}}$ is the signal energy per bit; $l_{k}$ path number of the $K^{\text {th }}$ user; $\mathrm{N}$ is the number of transmitted data bits; $h_{k, l}$ is attenuation factor for the $l^{\text {th }}$ path of the $K^{t h}$ user; $\mathrm{b}_{\mathrm{k}, \mathrm{m}}$ is the $m^{t h}$ data bit of the $K^{t h}$ user; $\mathrm{C}_{\mathrm{k}}(\mathrm{t})$ is the $\mathrm{PN}$ sequence of the $K^{\text {th }}$ user; $\mathrm{T}_{\mathrm{b}}$ is the bit period; $\mathrm{T}_{\mathrm{c}}$ is the PN sequence chip period; $\tau_{k, l}$ is the delay of $l^{\text {th }}$ path of the $K^{\text {th }}$ user.

\section{B. Middleton Class $A$ Model}

It refers to impulsive noise with a spectrum that is narrow compared to the receiver bandwidth and includes all pulses which do not produce transients in the receiver front end [15]. Its probability density function (pdf), given by:

$$
f_{x}(x)=e^{-A} \sum_{m=0}^{\infty} \frac{A^{m}}{m ! \sqrt{2 \pi \sigma_{m}^{2}}} e^{-\frac{x^{2}}{2 \sigma_{m}^{2}}}
$$

where

$$
\sigma_{m}^{2}=\frac{\frac{m}{A}+\Gamma}{1+\Gamma}
$$

is noise variance, $A=v_{t} T_{s}$ is impulse index, $v_{t}$ is mean impulse rate and $T_{s}$ is mean impulse duration. Equation (3) is a weighted sum of Gaussian distributions. By increasing impulse index, $A$, the noise can be made arbitrarily close to Gaussian and by decreasing Ait can be made arbitrarily close to a conventional Poisson process. The model assumes that the individual impulses are Poisson distributed in time. The scale factor $\Gamma$ is the ratio of powers in the Gaussian and Poisson (non-Gaussian) components, i.e.:

$$
\Gamma=\frac{\left(X_{G}^{2}\right)}{\left(X_{P}^{2}\right)}
$$

Fig.1 shows the pdf of Middleton class $A$ noise with various values of $A$ for $\Gamma=0.001$.

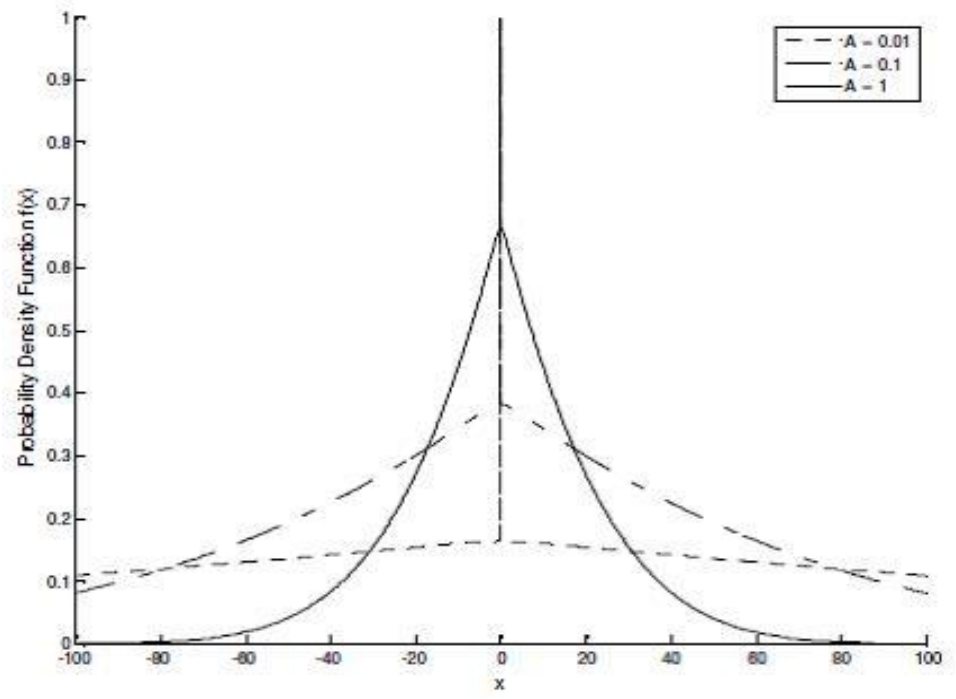

Figure (1): Probability density of the amplitude of Class-A noise with different values of A and $\Gamma=0.001[16]$ 


\section{BLIND SEPARATION OF DS-CDMA SIGNALS WITH ICA}

The received CDMA signal over multipath fading channel and additive impulse noise can be written as:

$$
r(t)=\sum_{k=1}^{K} \sum_{l=1}^{l_{k}} \sum_{m=1}^{N} \sqrt{\xi_{k}} b_{k, m} h_{k, l} C_{k}\left(t-m T_{b}-\tau_{k, l}\right)+n(t)
$$

where $n(t)$ is the class A impulsive noise.

This DS-CDMA system is an asynchronous system, that is to say $\tau_{k, l}$ is different for each user and path. But we assume that the length of $\mathrm{PN}$ sequence of each user is equal to $\mathrm{P}, \mathrm{T}_{\mathrm{c}}$ and $\mathrm{T}_{\mathrm{b}}$ are known and have the relationship of $\mathrm{T}_{\mathrm{b}}=\mathrm{PT}_{\mathrm{c}}$. Samples are taken from the observed data in $\mathrm{T}_{\mathrm{c}}$ cycle.

Define a sampled data vector $\boldsymbol{r}_{m}$ of length $2 P \times 1$,

$$
\boldsymbol{r}_{m}=\left[\begin{array}{llll}
r(t-(m-1) P+1) & r(t-(m-1) P+2) & \ldots & r(t-(m+1) P)
\end{array}\right]^{T}
$$

For asynchronous DS-CDMA system $\boldsymbol{r}_{m}$ can be written as:

$$
\boldsymbol{r}_{m}=\sum_{k=1}^{K} \sqrt{\xi_{k}}\left\{b_{k, m-1} \sum_{l=1}^{l_{k}} h_{k, l} \boldsymbol{g}_{k l}^{L}+b_{k, m} \sum_{l=1}^{l_{k}} h_{k, l} \boldsymbol{g}_{k l}^{C}+b_{k, l} \sum_{l=1}^{l_{k}} h_{k, l} \boldsymbol{g}_{k l}^{N}\right\}+\boldsymbol{n}(t)
$$

where $\mathbf{g}_{k l}^{L}$ is $2 P \times 1$ vector contains last $\tau_{k l}$ chips of the $K^{t h} \mathrm{PN}$ sequence; $\mathbf{g}_{k}^{C}$ is $2 P \times 1$ vector contains a complete period of the $K^{t h} \mathrm{PN}$ sequence; $\mathbf{g}_{k}^{F}$ is $2 P \times 1$ vector contains first $(P-$ $\tau k l$ chips of the $K t h$ PN sequence; $\boldsymbol{n} t$ is the $2 P \times 1$ vector of impulsive noise samples.

$$
\begin{aligned}
& \mathbf{g}_{k l}^{L}=\left[\begin{array}{lllllll}
C_{k}\left(P-\tau_{k l}\right. & \ldots & C_{k}(P) & 0 & \ldots & 0 & \\
+1)
\end{array}\right.
\end{aligned}
$$

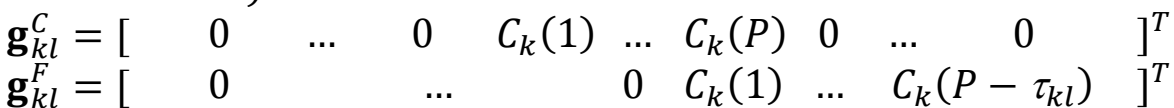

Fig.2 shows that the received signal $\boldsymbol{r}_{m}$ which must contain the complete periods of the three users codes and a beginning and ending of the three codes.

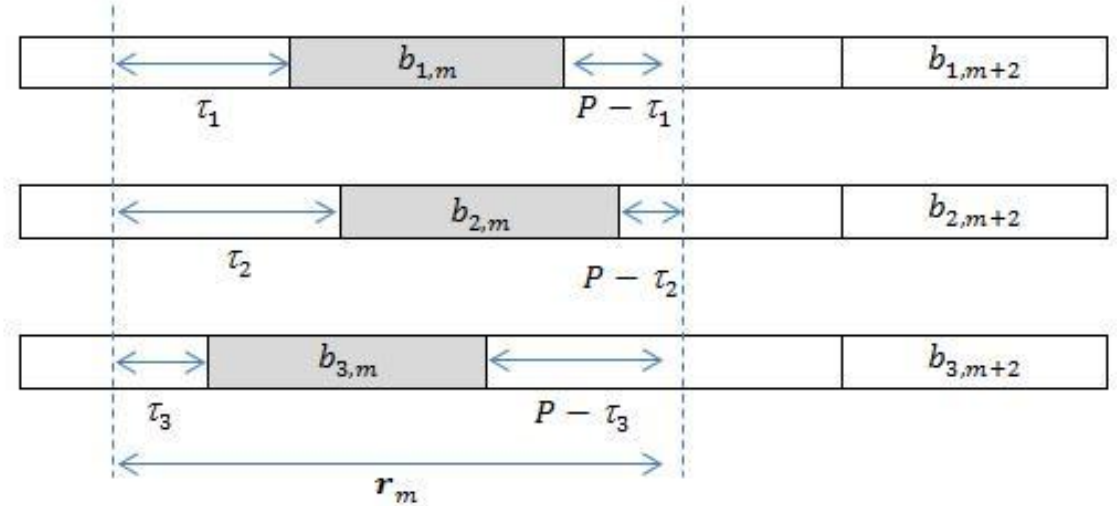

Figure (2): Sketch map of $\boldsymbol{r}_{m}$

If we want to express (9) in a matrix notation, we can replace $\mathrm{g}_{k l}^{\mathrm{L}}, \mathrm{g}_{k l}^{\mathrm{C}}$ and $\mathrm{g}_{k l}^{\mathrm{F}}$ with a matrix $\mathrm{G}$ and can be written as:

$$
\boldsymbol{Q}=\boldsymbol{G} \boldsymbol{B}+\boldsymbol{N}_{m}
$$


If the number of observed bits is N, we can define $\boldsymbol{Q}=\left[\boldsymbol{r}_{1}, \boldsymbol{r}_{2}, \ldots, \boldsymbol{r}_{N}\right]$ with dimension $2 \mathrm{C} \times \mathrm{N}$ and $\boldsymbol{B}=\left[\boldsymbol{b}_{1}, \boldsymbol{b}_{2}, \ldots, \boldsymbol{b}_{N}\right]$ with dimension $3 \mathrm{~K} \times \mathrm{N}$ and $\boldsymbol{b}_{m}$ is $3 \mathrm{~K} \times 1$ vector composed of the $3 \mathrm{~K}$ $\begin{array}{lllll}\text { statistically independent data bit with } & \boldsymbol{b}_{m}=\end{array}$ $[b 1, m-1, b 1, m, \quad b 1, m+1, \ldots, b k, m-1, b k, m, b k, m+1] T$ with dimension and $\boldsymbol{G}=\left[\boldsymbol{g}_{1 l}^{L}, \boldsymbol{g}_{1 l}^{C}, \boldsymbol{g}_{1 l}^{F}, \ldots, \boldsymbol{g}_{k l}^{L}, \boldsymbol{g}_{k l}^{C}, \boldsymbol{g}_{k l}^{F}\right]$ with dimension $2 \mathrm{C} \times 3 \mathrm{~K}$.

In the regular ICA process, the observed data should be preprocessed, including centering and whitening.

\section{A. Centering}

The received baseband signal $r(t)$ is centered by removing its mean,[16]

$$
r_{c}(t)=r(t)-E[r(t)]
$$

B. Whitening

Whiteness of a Zero mean random vector means that its components are uncorrelated and their variances equal unity. Whitening using the principal component analysis (PCA) method can reduce dimension and make ICA process more accurate and faster. The whitening method isas follows:

Eigen value decomposition (EVD) of the covariance matrix $R$ of the sampled observations are:

$$
\boldsymbol{R}=\boldsymbol{U} \boldsymbol{D} \boldsymbol{U}^{T}=\boldsymbol{G} \boldsymbol{G}^{T}+\sigma_{n}^{2} \mathbf{I}=\left[\begin{array}{ll}
\boldsymbol{U}_{S} & \boldsymbol{U}_{N}
\end{array}\right]\left[\begin{array}{cc}
\lambda_{S}+\sigma_{n}^{2} \mathbf{I} & 0 \\
0 & \sigma_{n}^{2} \mathbf{I}
\end{array}\right]\left[\begin{array}{l}
\boldsymbol{U}_{S}^{T} \\
\boldsymbol{U}_{N}^{T}
\end{array}\right]
$$

where $\boldsymbol{U}_{S}$ is the signal subspace with dimension of $2 P \times 3 K ; \boldsymbol{U}_{N}$ is the noise subspace with dimension of $2 P \times(2 P-3 K) ; \lambda_{S}$ is the diagonal matrix of eigenvalues of the signal subspace; $\sigma_{n}^{2}$ is the variance of noise.

Then whitening is applied by projecting to the signal subspace so the correlation of different user's signal could be removed. The whitening matrix is:

$$
\boldsymbol{V}=\left(\boldsymbol{\lambda}_{S}+\sigma_{n}^{2} \mathbf{I}\right)^{-1 / 2} \boldsymbol{U}_{S}^{T}
$$

The data after whitening is:

$$
\begin{gathered}
\boldsymbol{Z}=\boldsymbol{V} \mathbf{Q}=(\boldsymbol{V} \boldsymbol{G}) \boldsymbol{B}+\boldsymbol{V} \boldsymbol{N}=\boldsymbol{A} \boldsymbol{B}+\boldsymbol{N}^{\prime} \\
\boldsymbol{A}=\boldsymbol{V} \boldsymbol{G}=\left(\boldsymbol{\lambda}_{S}+\sigma_{n}^{2} \mathbf{I}\right)^{-1 / 2} \boldsymbol{U}_{S}^{T} \boldsymbol{G}
\end{gathered}
$$

A is the mixing matrix which is a full rank and square matrix. The dimension of the signal after whitening is reduced from $2 P$ to $3 K$. Using ICA method, an orthogonal matrix $\boldsymbol{W}=\boldsymbol{A}^{-1}$ can be found to estimate the data $\mathbf{B}$.

$$
\widehat{B}=W Z
$$

C. ICA algorithm 
Through the preprocessing steps above, data $Z$ are ready to be processed by ICA. The fastICA algorithm is based on a fixed point iteration scheme for finding the maximum of the noneGaussianity[17]. From the information theory, it is rational to use negentropy as the measurement of the non-Gaussianity of stochastic variables. In [18], two none linear functions $f$ are recommended to estimate the negentropy.

$$
f_{1}(u)=u^{3}, f_{2}(u)=u \exp \left(-u^{2} / 2\right)
$$

ICA based on the negentropy criterion achieved the separation of the signal through following steps:

1. Choose $m$, the number of ICs to estimate. Set $l \leftarrow 1$.

2. Initialize $\boldsymbol{w}_{l}$ (e.g. randomly)

3. Do an iteration of a one-unit algorithm on $\boldsymbol{w}_{l}$.

$$
\boldsymbol{w}_{l} \leftarrow \frac{1}{N} \sum_{m=1}^{N}\left[\boldsymbol{z}_{m}\left(\boldsymbol{w}_{l}^{T} \mathbf{z}_{m}\right)^{3}\right]-3 \boldsymbol{w}_{l}
$$

4. Do the following orthogonalization:

$$
\boldsymbol{w}_{l} \leftarrow \boldsymbol{w}_{l}-\sum_{j=1}^{l-1}\left(\boldsymbol{w}_{l}^{T} \boldsymbol{w}_{j}\right) \boldsymbol{w}_{j}
$$

5. Normalize $\boldsymbol{w}_{l}$ by dividing it by its norm.

6. If $\boldsymbol{w}_{l}$ not converged, i.e. $\left|\boldsymbol{w}_{l}^{T} \boldsymbol{w}_{l-1}\right|$ is not close enough to 1 , go back to 3 .

7. Set $l \leftarrow l+1$. If $l$ is not greater than the desired number of ICs, go back to step 2 .

When iteration ended, the unmixing matrix $\mathbf{W} \approx \mathbf{A}^{-1}$ is calculated out. When left multiplied by $\mathbf{W}$ and right multiplied by the matrix of pseudo random sequences, $\mathbf{G}^{\mathrm{T}}$, expression (21)can be written as:

$$
\mathbf{W} \cdot\left(\boldsymbol{\lambda}_{\mathrm{S}}+\sigma_{\mathrm{n}}^{2} \mathbf{I}\right)^{-1 / 2} \mathbf{U}_{\mathrm{S}}^{\mathrm{T}} \mathbf{G G}^{\mathrm{T}}=\mathbf{G}^{\mathrm{T}}
$$

From expression (18), $\mathbf{G}^{\mathrm{T}} \approx \mathbf{U}_{\mathrm{S}}\left(\boldsymbol{\lambda}_{\mathrm{S}}+\sigma_{\mathrm{n}}^{2} \mathbf{I}\right) \mathbf{U}_{\mathrm{S}}^{\mathrm{T}}$, thus the matrix of pseudo random sequence could be estimated by :

$$
\begin{gathered}
\mathbf{G}^{\mathrm{T}}=\mathbf{W} \cdot\left(\boldsymbol{\lambda}_{\mathrm{S}}+\sigma_{\mathrm{n}}^{2} \mathbf{I}\right)^{-\frac{1}{2}} \mathbf{U}_{\mathrm{S}}^{\mathrm{T}} \mathbf{U}_{\mathrm{S}}\left(\boldsymbol{\lambda}_{\mathrm{S}}+\sigma_{\mathrm{n}}^{2} \mathbf{I}\right) \mathbf{U}_{\mathrm{S}}^{\mathrm{T}} \\
\mathbf{G}=\left(\mathbf{W} \cdot\left(\boldsymbol{\lambda}_{\mathrm{S}}+\sigma_{\mathrm{n}}^{2} \mathbf{I}\right)^{\frac{1}{2}} \mathbf{U}_{\mathrm{S}}^{\mathrm{T}}\right)^{T}
\end{gathered}
$$

From the expression above, the pseudo random sequence could be recovered directly.

\section{TRIPLE CORRELATION FUNCTION}

TCF method utilized the shift-and-add property of m-sequence [19],

$$
M_{0} \oplus M_{D}=M_{E}
$$

where $M_{0}$ is the original m-sequence, $M_{D}$ is the shift form of $M_{0}$ with D chips delay, $M_{E}$ is another shift form of $M_{0}$ with E chips delay.

The triple correlation function is a $3^{\text {rd }}$ order statistic in fact which is defined: 


$$
R_{3, x}\left(\tau_{1}, \tau_{2}\right)=E\left\{x(t) x\left(t+\tau_{1}\right) x\left(t+\tau_{2}\right)\right\}
$$

for m-sequences, let $\tau_{1}=p T_{c}, \tau_{2}=q T_{c}$, the TCF of $\mathrm{m}$-sequence could be written as[6]:

$$
R_{3, x}(p, q)=E\{x(n) x(n+p) x(n+q)\}
$$

let $x(n)$ be $\mathrm{M}_{0}, x(n+p)$ be $\mathrm{M}_{\mathrm{p}}$, and $x(n+q)$ be $\mathrm{M}_{\mathrm{q}}, E\{x(n) x(n+p)\}$ is equal to a shift form of $x(n)$ with $\mathrm{v}$ chips delay, supposing the shift form is $\mathrm{M}_{\mathrm{v}}$, then TCF could be written as:

$$
R_{3, x}(p, q)=E\left\{x_{v} \cdot x_{q}\right\}=R_{x x}(v, q)
$$

Expression (28) is the autocorrelation function of $x(n+v)$ and $x(n+q)$, according to the autocorrelation character of $\mathrm{m}$-sequence, if and only if $v=q, R x x(v, q)$ reached its maximum, for other $v \neq q, R x x(v, q)$ is minimum. The TCF of $\mathrm{m}$-sequence is of three degrees, when plotted in $x-y-z$ coordinate system, the TCF is something like peaks erected above the ground and the pattern of the peaks is uniquely determined by the primitive polynomial of the $\mathrm{m}$-sequence. Therefore, from the pattern of TCF, the primitive polynomial of m-sequence could be uniquely determined.

In the situation of multipath, data recovered by ICA are the overlapped pseudo random sequence of different paths. Let the $k^{t h}$ estimated data is $x_{k}(t)=w_{k}(t)+v_{k}(t), w_{k}(t)$ is the overlap pseudo random sequence in the $k^{t h}$ degree and $v_{k}(t)$ is noise in the same degree. According to the additive character of high order statistic, when the order is lager than 2, the HOS value of Gaussian stochastic process tended to be zero, the TCF of $x_{k}(t)$ is

$$
R_{3, x_{k}}(p, q)=R_{3, w_{k}}(p, q)+R_{3, v_{k}}(p, q) \sim \sum_{l=1}^{L_{k}} h_{k l}^{3} R_{3, C_{k l}}(p, q)
$$

Two paths of $C_{k i}$ and $C_{k j}$ are mainly considered here, other paths could be analyzed through the same way.

$$
R_{3, C_{k i}}(p, q)=\frac{1}{N} \sum_{n=1}^{N} h_{k i}^{3} C_{k i}(n) C_{k i}(n+p) C_{k i}(n+q)
$$

Where $C_{k i}$ is the $d_{k i}$ chips delay form of the $K^{\text {th }}$ pseudo random sequence $C_{k}$, $\Sigma C_{k i}(\mathrm{n}) C_{k i}(\mathrm{n}+\mathrm{q})$ could be considered as a shift form of $C_{k}$ with D chips delay. If and only if $q=D, R_{3, C_{k i}}(p, q)$ (the autocorrelation function of $C$ ) reached its maximum, for other $q \neq D$, $R_{3, C_{k i}}(p, q)$ is the minimum. For $C_{k j}$,

$$
R_{3, C_{k j}}(p, q)=\frac{1}{N} \sum_{n=1}^{N} h_{k j}^{3} C_{k j}(n) C_{k j}(n+p) C_{k j}(n+q)
$$

$\Sigma C_{k j}(\mathrm{~m}) C_{k j}(\mathrm{~m}+\mathrm{q})$ could be considered as a shift form of $C_{k}$ with E chips delay. Though $\mathrm{m}=\bmod \left(\mathrm{n}+d_{k i}-d_{k j}, \mathrm{P}\right)$ is different from $\mathrm{n}, \mathrm{p}$ did not change, so the point $q=D$ is still the maximum of $R_{3, C_{k j}}(p, q)$ )That is, TCF could eliminate the influence of multipath.

\section{SIMULATION}

In order to test the performance of the method proposed above, several experiments are performed in this section. The simulation parameters are as follows: DS-CDMA signal, BPSK modulation, the number of bits is $10^{5} \mathrm{bits}$, the $\mathrm{PN}$ sequence is $\mathrm{m}$-sequence with the length of 63 , the SNR is $-10 \mathrm{~dB}$, there are three users, the signals are assumed to transmitted over 
typical urban channel with six taps with maximum delay spread $\sigma_{T}=0.5 \times 10^{-5}$ and contaminated with Class-A impulsive noise.

Now we estimate the PN sequence of each user using the method proposed above. Fig. 3 shows the original PN sequence waveforms of the 3 users, the first user has a m-sequence of a polynomial $P(x)=x^{6}+1$, the second user has a m-sequence of polynomial $P(x)=x^{6}+$ $x^{5}+x^{2}+x+1$ and the third user has a m-sequence of polynomial $P(x)=x^{6}+x^{5}+x^{3}+$ $x^{2}+1$. Fig. 4 shows time realization of highly impulsive $\left(A=0.1\right.$ and $\left.\Gamma=0.5 \times 10^{-3}\right)$ Middleton class A impulsive noise. Fig.5 shows the signal of each user under multipath channels and the total received signal at the receiver under class-A impulsive noise with $\mathrm{SNR}=-10 \mathrm{~dB}$. Fig. 6 exhibits the TCF of the overlapped estimated data in $x-y-z$ coordinate system. Though there are some noise swell above the ground, the peaks still erect up straightly. Fig. 7 is the TCF of true pseudo random sequence of the same user. From Fig. 6 and Fig. 7, the pattern of peaks is completely the same, so the prime polynomial could be estimated easily from TCF. Fig. 8 shows the relationship between the correct estimation number of pseudo random sequence and SNR. The data of Fig. 8 is the average result of 100 Monte Carlo experiments. The number of users three and the channel is typical urban channel with six taps and Rayleigh distribution, this figure shows that the performance of the estimated codes for the three users are almost the same for different SNR and shows that the estimation reach for $100 \%$ for $-8 \mathrm{~dB}$ SNR .
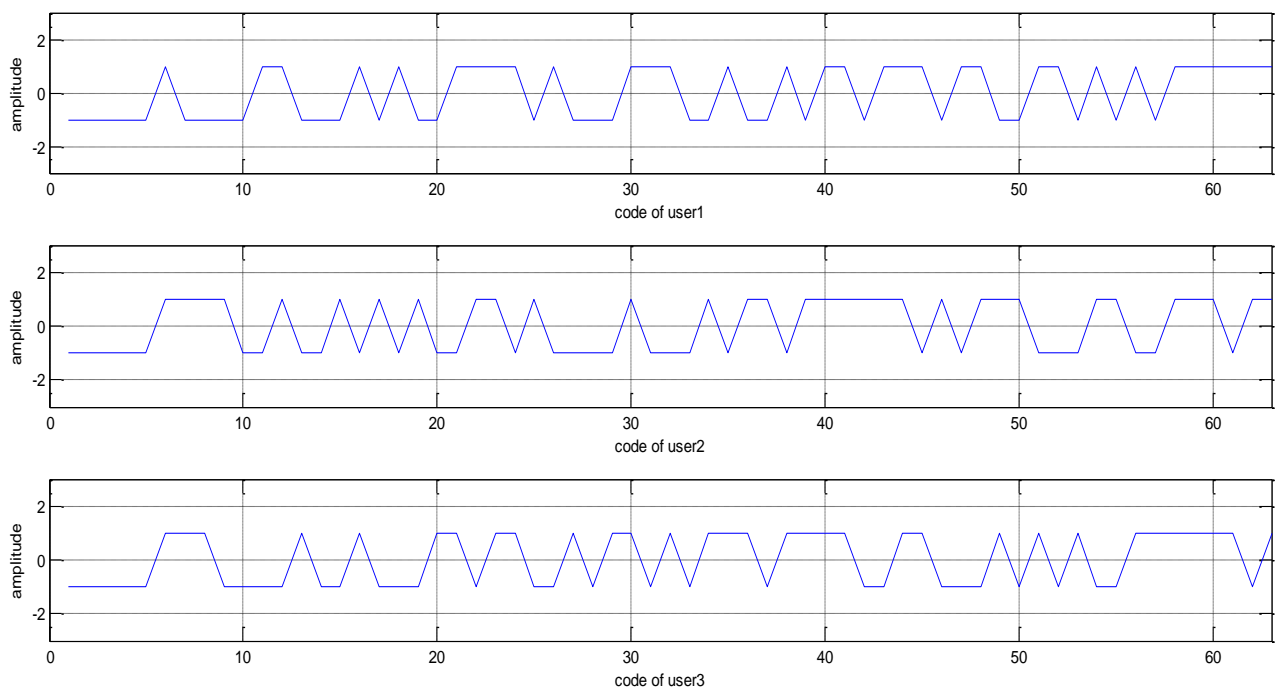

Figure (3): The original PN sequence waveforms of the 3 users 


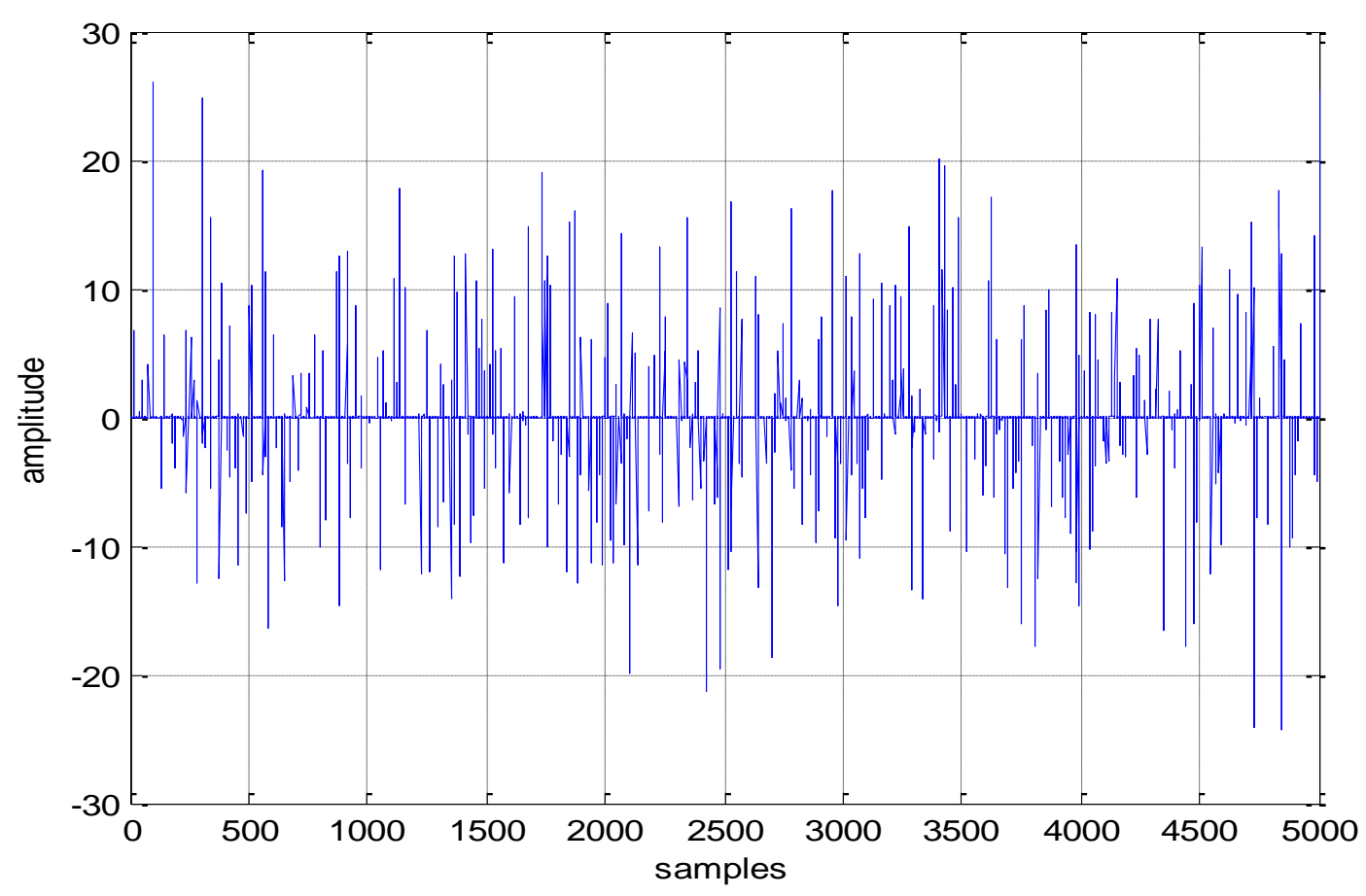

Figure (4): Time realization of highly impulsive $\left(\mathrm{A}=0.1\right.$ and $\left.\Gamma=0.5 \times 10^{-3}\right)$ Middleton class A noise
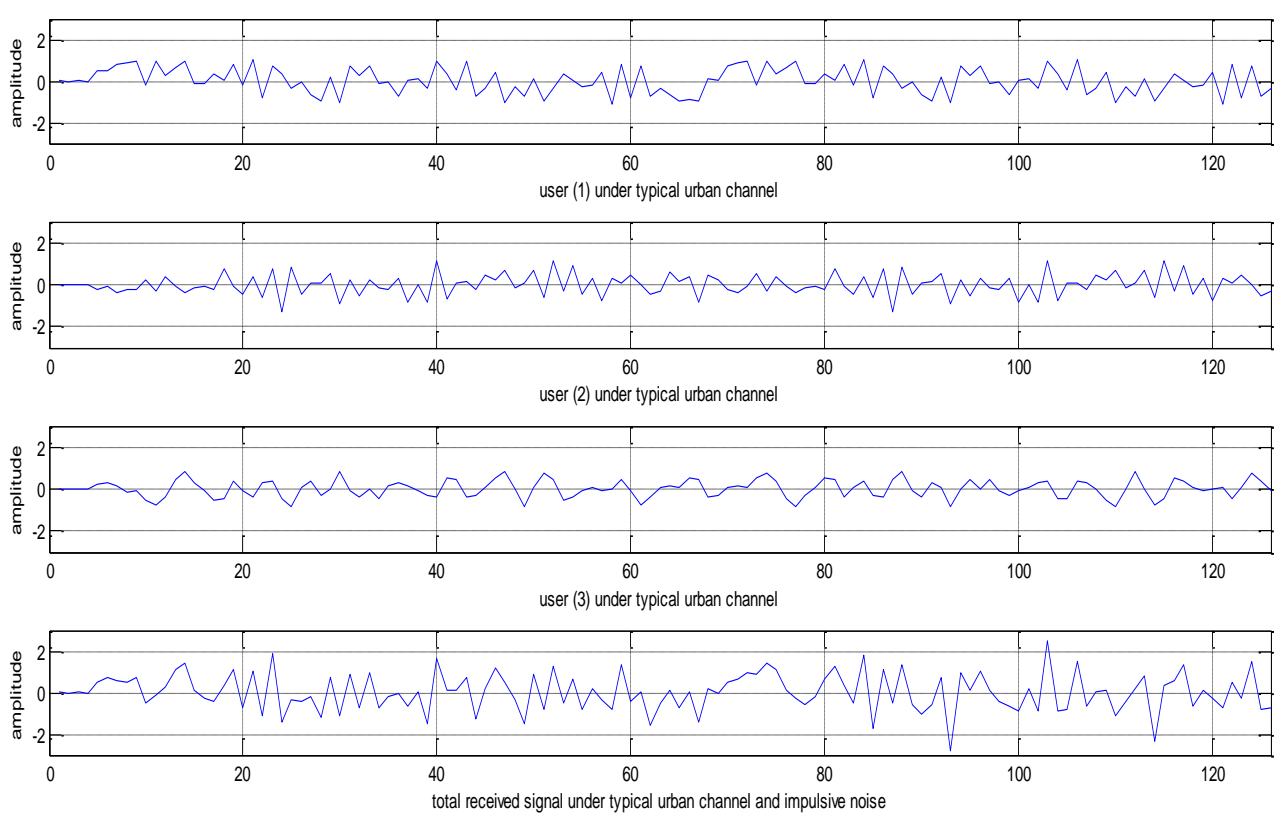

Figure (5): The total received data of the three users under impulsive noise and multipath channel 


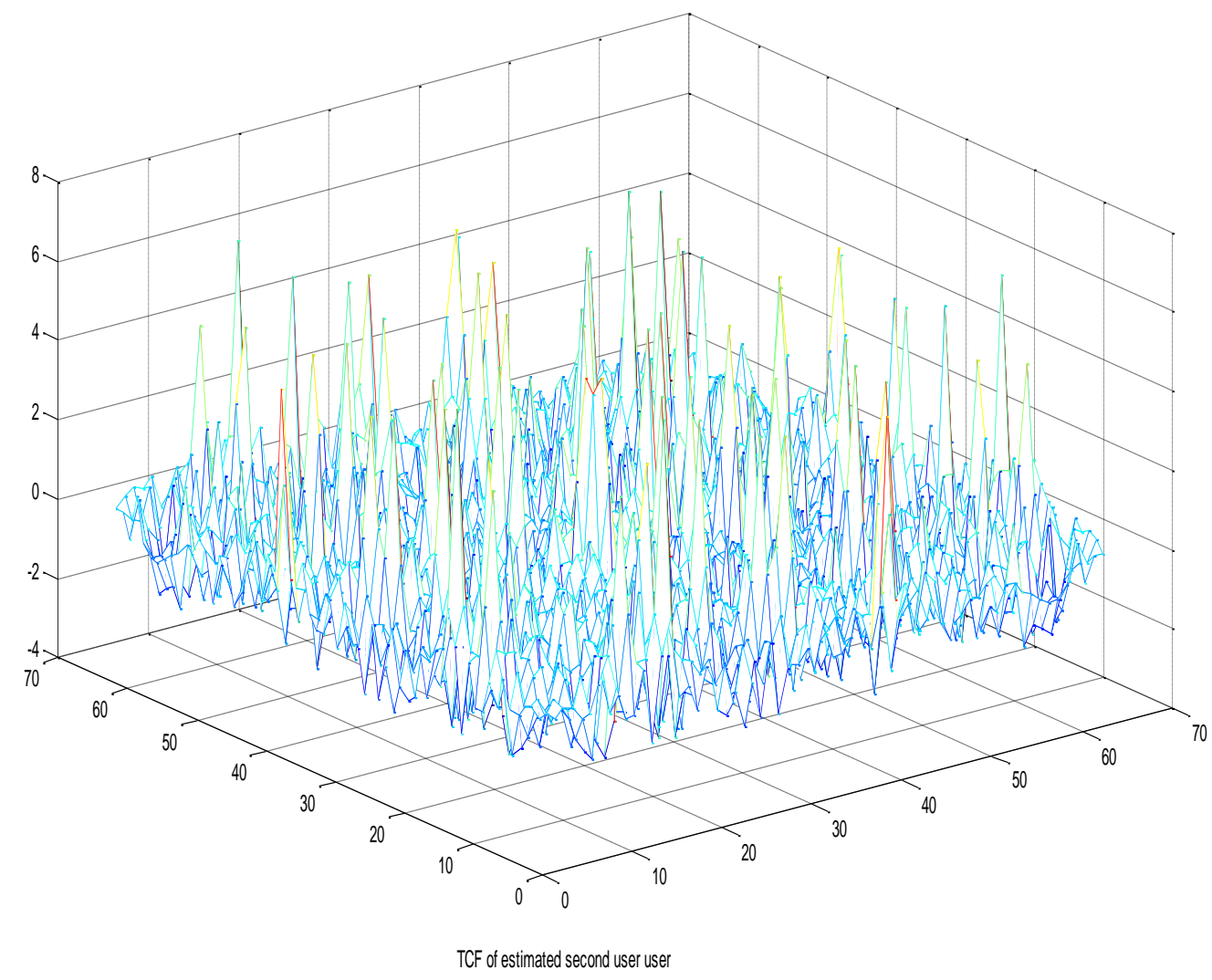

Figure (6): TCF of the estimated second user code

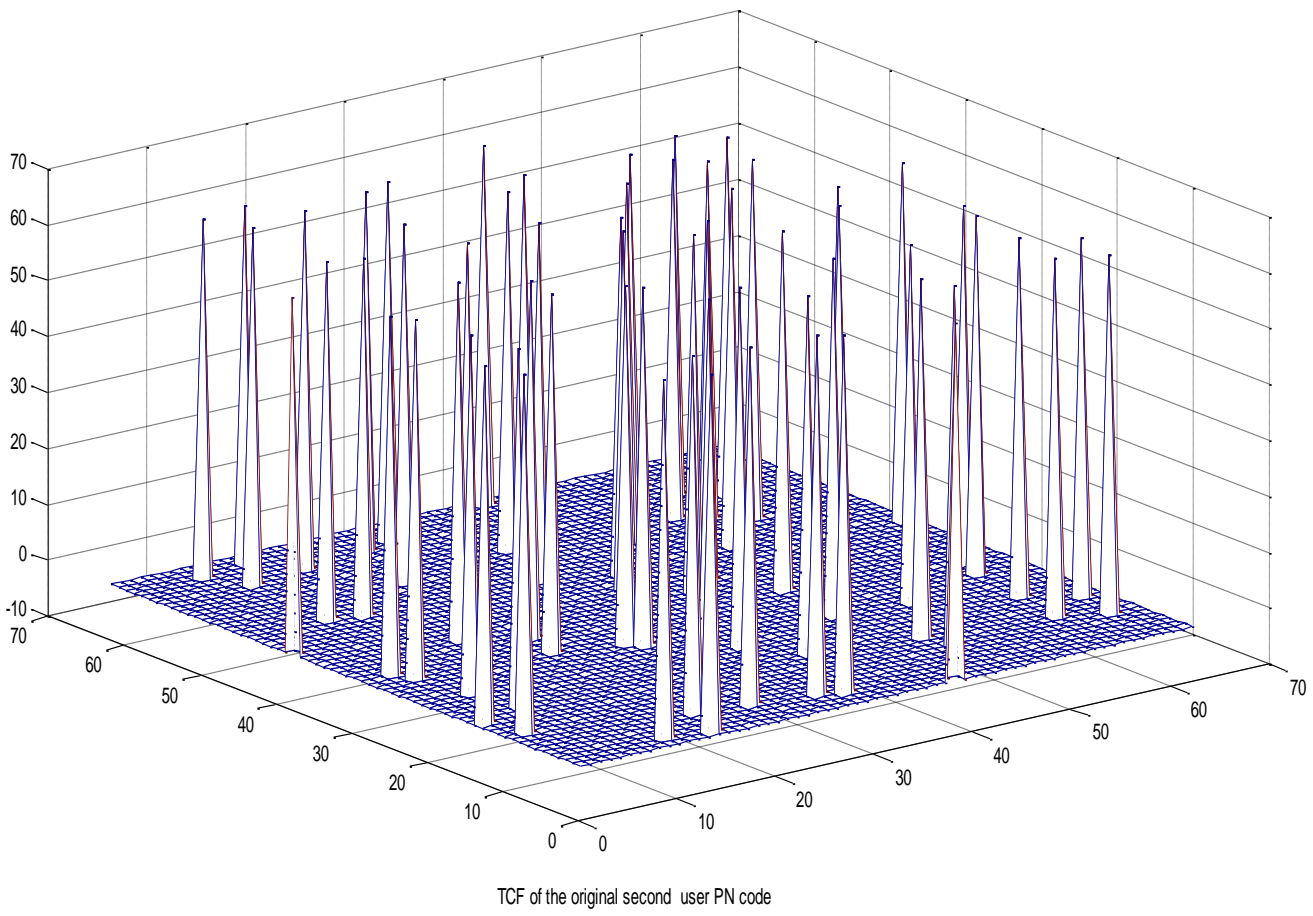

Figure (7): TCF of the original second user PN code 


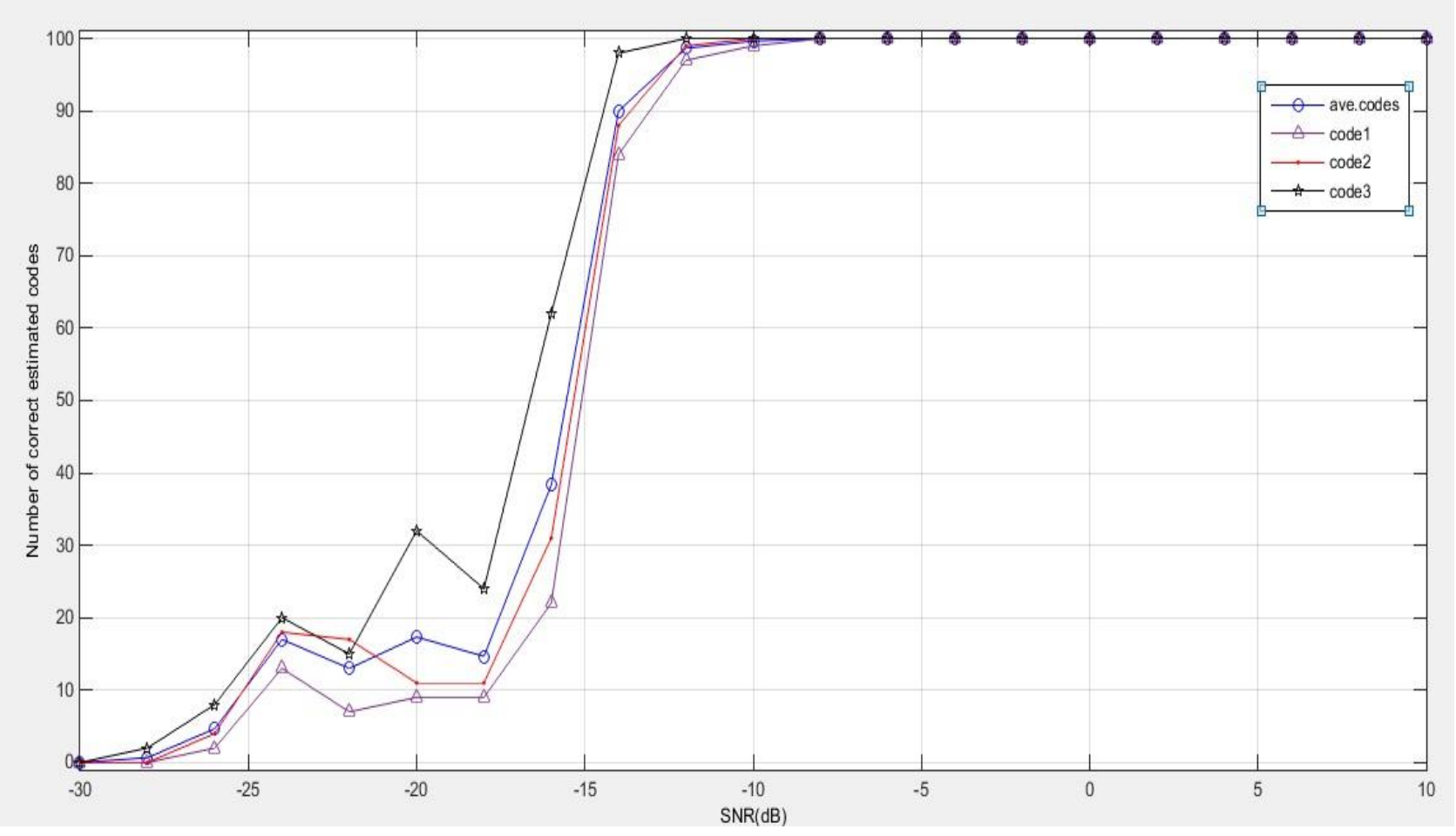

Figure 8: The number of correct estimated codes three codes at $\mathrm{SNR}=-13 \mathrm{~dB}$

\section{Conclusion}

Technology ICA could be used to achieve the blind separation of DS-CDMA signals. When the subspace of pseudo random sequence is multiplied by the unmixing matrix which is calculated from ICA, the estimated waveform of pseudo random could be acquired. When there is only one path for each user, the estimated waveform is the accurate estimation of the pseudo random sequence. But when there are two or more paths for each user, the waveform is the overlapped form of the pseudo random sequences with different path delays. TCF is a three order statistic base on the logic character of $\mathrm{m}$-sequence, from the pattern of TCF, the prime polynomial could be identified uniquely. So, when m-sequences are used as pseudo random sequences, processed by TCF, the estimated correct rate of pseudo random sequences with ICA method is increased greatly.

In this paper, the DS-CDMA signal model under impulsive noise and multipath fading channel is presented; we also give the theoretical derivation and computer simulation. The proposed method can correct estimate the PN sequences of each user in low SNR.

\section{References}

[1] Yongpeng Wei, Luokun Liu, Jian Zhang "Blind Estimation of PN Sequence of DSCDMA Signal in Multipath" 2012 IEEE 2nd International Conference on 978-1-4577$1415-3 / 12 / \$ 26.00$

[2] J.Massey, "Shift register synthesis and BCH decoding", IEEE transactions on Information Theory,1969,15(1):122-127

[3] P. Hill, M. Ridley, "Blind Estimation of direct-sequence spread spectrum m sequence chip codes," IEEE sixth International Symposium on Spread Spectrum Techniques and Applications, 2000, pp. 305-309.

[4] G. Burel, C. Bouder. "Blind estimation of the pseudo-random sequence of a direct sequence spread spectrum signal,” IEEE Milcom 2000, pp. 967-970. 
[5] C.Nzoza, R.Gautier, G.Burel, "Blind synchronization and sequences identification in CDMA transmissions", IEEE MILCOM 2004, vol.3.

[6] C.Bouder, S. Azou, G.Burel,"Performance analysis of a spreading sequence estimator for spread spectrum transmissions",Journal of theFranklin Institute 341(2004), pp:595614.

[7] O.Ekici, A.Yongacoglu, Application of noisy-independent component analysis for CDMA signal separation, VTC-Fall,2004,Vol.5.

[8] D.Hill, J. Bodie, Carrier detection of PSK signals. IEEE Trans. On Communications 2001,49(3):487-496.

[9] J.Yan,J.Hongbing, "A cyclic-cumulant based method for DS-SS signal detection and parameter estimation",IEEE International Symposium on Microwave, Antenna, Propagation and EMC Technologies for Wireless Communications,2005, vol2.

[10] G.Burel, "Detection of spread spectrum transmissions uing fluctuations of correlation estimators", IEEE ISPACA,2000

[11] T.Ristaniemi, J.Joutsensalo, "Advanced ICA-Based receivers for block fading DSCDMA channels," Signal Processing, 82, pp. 417-431, 2002.

[12] Simon, M.K., Omura J.K., Scholtz R.A., Levitt B.K., Spread Spectrum Communications Handbook, McGraw- Hill, 2002.

[13] Glover, Q. Shan, P. J. Mooore, I. E. Portugues, and R. J. Watson "An Investigation into the Vulnerability of WLAN and WPAN Technologies to Impulsive Noise in Electricity Transmission Substations," Ninth International Symposium on Communication Theory and Applications ISCTA'07, Ambleside, Lake District, UK, 2007.

[14] M.G. Sanchez, I Cuinas, and A. V. Alejos, "Interference and Impairments in Radio Communication Systems due to Industrial Shot Noise, "IEEE International Symposium on Industrial Electronics ISIE 2007, PP1849-1854.

[15] Middleton, "Canonical and Quasi-Canonical Probability Models of Class a Interference," Electromagnetic Compatibility, IEEE Transactions on, vol. EMC-25, pp. 76-106, 1983.

[16] Miao Yu, Shiju Li , Lei Shen "Blind Estimation of Pseudo Random Sequence of DS-CDMA Signals with ICA Method” 978-1-4244-5895-0/10/\$26.00 @2010 IEEE

[17] Hyvarinen, E. Oja, "A fast fixed-point algorithm for independent component analysis," Neural Computation, 1997, 9(7), pp. 1483-1492.

[18] Hyvarinen, E. Oja, "Independent component analysis algorithms and application," IEEE Trans.Neural Networks, 2000, pp. 411-430.

[19] M.K. Simon, J.K. Omura, R.A. Scholtz, et al., Spread spectrum communications handbook, McGraw-Hill,1994, New York, US. 